\title{
OPEN The effect of age-related macular degeneration on cognitive test performance
}

\begin{abstract}
Anne Macnamara ${ }^{1 凶}$, Victor R. Schinazi ${ }^{2,3}{ }^{,}$Celia Chen $^{4}$, Scott Coussens $^{1} \&$ Tobias Loetscher $^{1}$
The reliable assessment of cognitive functioning is critical to the study of brain-behaviour relationships. Yet conditions that are synchronous which ageing, including visual decline, are easily overlooked when interpreting cognitive test scores. The purpose of this study was to demonstrate the negative consequences of visual impairments on cognitive tests performance. Moderate to severe levels of age-related macular degeneration were simulated, with a set of goggles, in a sample of twenty-four normally sighted participants while they completed two cognitive tasks: a vision-dependent reaction time task and a vision-independent verbal fluency test. Performance on the reaction time task significantly decreased $(p<0.001)$ in the simulated age-related macular degeneration condition, by as much as 25 percentile ranks. In contrast, performance on the verbal fluency test were not statistically different between the simulated and normal vision conditions $(p=0.78)$. The findings highlight the importance of considering visual functioning when assessing cognitive function. When vision is not accounted for, low test scores may inaccurately indicate poor cognition. Such false attributions may have significant ramification for diagnosis and research on cognitive functioning.
\end{abstract}

Cognitive tests scores inform research and diagnoses in aging and neurodegenerative disorders. However, these scores can be impacted by a range of factors not directly measured by tests, ranging from situational, personal, language to cultural factors ${ }^{1}$. While some of these factors may be easier to identify, others are more elusive. For example, a language barrier can become quickly apparent if participants struggle to understand instructions or perform adequately on a written task. On the other hand, impairments of a visual nature can be harder to recognise as there may be no clear indication of impaired visual function ${ }^{2}$. Indeed, vision impairments are often overlooked in research and clinical settings; it has previously been estimated that reduced vision may be undetected in up to $50 \%$ of older adults ${ }^{3}$.

In 2020, moderate to severe vision impairment affected approximately 200 million people over the age of $50^{4}$. Given that the prevalence of visual impairment is only estimated to increase due to the aging population, researchers and clinicians focusing on ageing and neurodegenerative disorders need to pay close attention to the possibility that visual impairments may affect the scores of cognitive tests. One leading cause of visual impairment is age-related macular degeneration (AMD), which may result in an irreversible loss of central vision ${ }^{5}$; and can negatively impact tasks involving visual functioning including reading, driving and recognising faces ${ }^{6}$. Critically, AMD is known to be underdiagnosed in the elderly, with an estimated $25 \%$ of eyes medically judged to be 'normal', actually having features of AMD and suboptimal vision'.

To highlight the importance of central vision for cognitive assessments, we simulated visual impairment with AMD simulation goggles, while participants completed a series of cognitive tasks. Since older adults with visual impairments are significantly more vulnerable to physical and mental comorbidities (i.e., Parkinson's disease, dementia, hearing loss), a vision loss simulation with healthy, normally-sighted participants can more easily isolate vision-related effects on behaviour ${ }^{8,9}$. While simulations may never wholly replicate a visual impairment (e.g., due to patient variability in symptom presentation; underdeveloped compensatory strategies; and lack of progressive visual decline $)^{9}$, thus far simulating vision loss has been a simple, yet valid approach to investigate the effects of visual impairments on cognition ${ }^{10,11}$. Furthermore, AMD simulations have replicated patterns of behaviour and difficulties experienced by AMD patients.

${ }^{1}$ Cognitive Ageing and Impairment Neurosciences Laboratory, Justice and Society, University of South Australia, Adelaide, SA, Australia. ${ }^{2}$ Department of Psychology, Faculty of Society and Design, Bond University, Gold Coast, QLD, Australia. ${ }^{3}$ Future Health Technologies, Singapore-ETH Centre, Campus for Research Excellence and Technological Enterprise (CREATE), Singapore, Singapore. "Department of Ophthalmology, Flinders Medical Centre, Flinders University, Adelaide, SA, Australia. ${ }^{\square}$ email: anne.macnamara@mymail.unisa.edu.au 


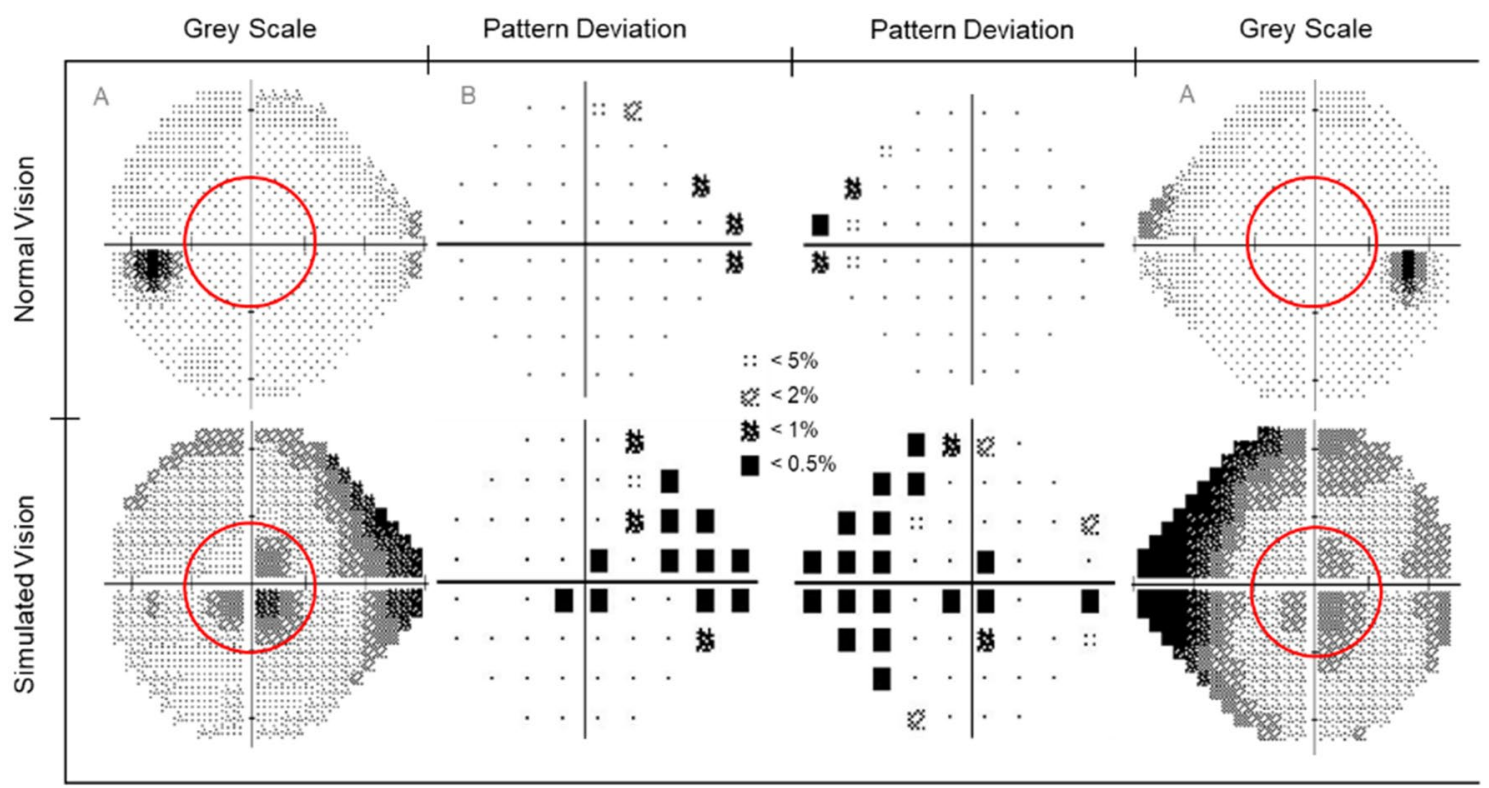

Figure 1. Results of the visual field test. Top row: normal visual field in the left and right eye. Bottom row: simulated macular degeneration visual field with a central $10^{\circ}$ scotoma (red circles).

\section{Methods}

Participants. An a priori power calculation was conducted to estimate the required number of participants for a larger study investigating the effects of simulated AMD on anxiety and stress levels in everyday activities (not reported here). Using $\mathrm{G}^{*}$ Power $^{12}$, it was estimated that a minimum of 13 participants were required to provide sufficient power ( 0.90 ), at a significance level of $\alpha=0.05$, to detect a large effect. A large effect could increase the practical significance of the findings. The estimate was based upon an AMD simulation study which was similar in nature to the larger study conducted ${ }^{13}$. All eligible participants that signed up during the advertisement period (between July and September 2020) were tested. Data were only analysed after data collection was completed.

Twenty-four normal-to-corrected sighted (best corrected visual acuity of greater $>6 / 18$ ) participants (19 women) aged 18-60 (Mean =27.1, SD =9.7) completed the experiment. They were English speakers, and had no history of visual impairment, anxiety disorders, psychiatric disorders, or cognitive impairment. Participants were recruited at the University of South Australia (UniSA) and via the UniSA online research participation system, and informed consent was obtained from all. The study was approved by the UniSA Human Research Ethics Committee (Ethics Protocol 202889); and it was conducted in accordance with the Declaration of Helsinki, and the Australian National Statement on Ethical Conduct in Human Research guidelines.

Apparatus and materials. The visual effect of AMD was induced with enhanced Fork in the Road Macular Degeneration simulator goggles ${ }^{14}$. The severity was manipulated to reflect moderate to severe AMD—visual acuity $6 / 18$ to $3 / 60$ respectively ${ }^{15}$. The goggles were enhanced by the addition of two layers of $20 \mathrm{~mm}$ diameter circular Bangerter occlusion foils of 0.1 LogUnit (resulting in 20/200 or 6/60 vision), positioned in the central inner region of each lens. These enhancements were calibrated by a neuro-ophthalmologist to ensure the simulator resulted in a reduction in best corrected visual acuity to $6 / 60$ and created a $10^{\circ}$ central scotoma monocularly in each eye. The visual acuity was verified using Snellen linear acuity at $6 \mathrm{~m}$ and the scotoma was confirmed with a Zeiss Humphry 24-2 automated visual field analyser (see Fig. 1; Carl Zeiss Meditec, Inc. Jena, Germany). To ensure the results were not confounded by the goggle frames (e.g., restricted peripheral vision), identical goggles with clear lenses were worn in the normal vision condition. If required, participants wore prescription glasses under the goggles.

Cognition was assessed via a vision-dependent Reaction Time Task (RTI ${ }^{16}$ and a vision-independent Verbal Fluency Test $(\mathrm{VFT})^{17}$. The tests were chosen as they are suitable for assessing cognition in aging, clinical populations (e.g., Alzheimer's) ${ }^{18-21}$. The RTI [choice], from the Cambridge Neuropsychological Test Automated Battery $(\mathrm{CANTAB})^{16}$, assesses mental and motor response speeds. Participants pressed a button on the screen, after which a yellow dot appeared in one of five circle locations. Participants were instructed to move their finger from the button to the yellow dot, as quickly and accurately as possible. Mental responses reflected the times taken for participants to identify the yellow dot location and release the button. Motor responses were the times taken for participants to move from the button to the yellow dot.

The VFT appraises semantic and phonemic fluency ${ }^{17}$. Participants had sixty seconds to generate as many different words (excluding names, places, and repeated words with different endings), starting with the letter $\mathrm{F}$ or S. The VFT was conducted as a control task, because unlike the RTI, it does not require vision for completion. 


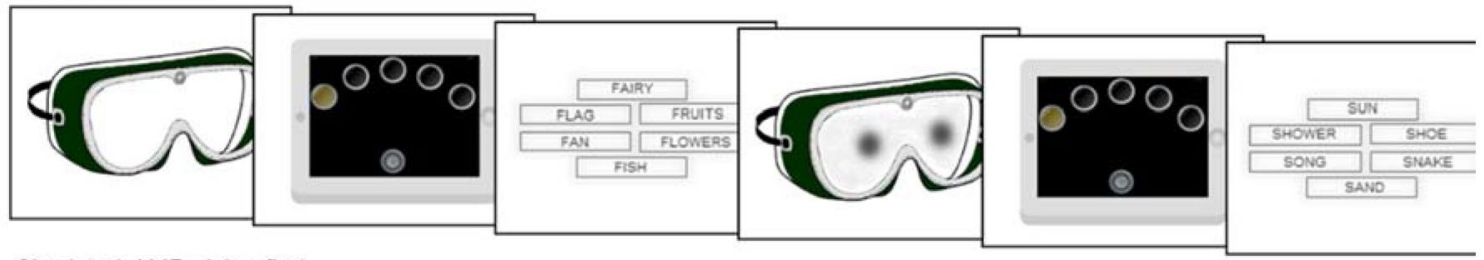

Simulated $A M D$ vision first

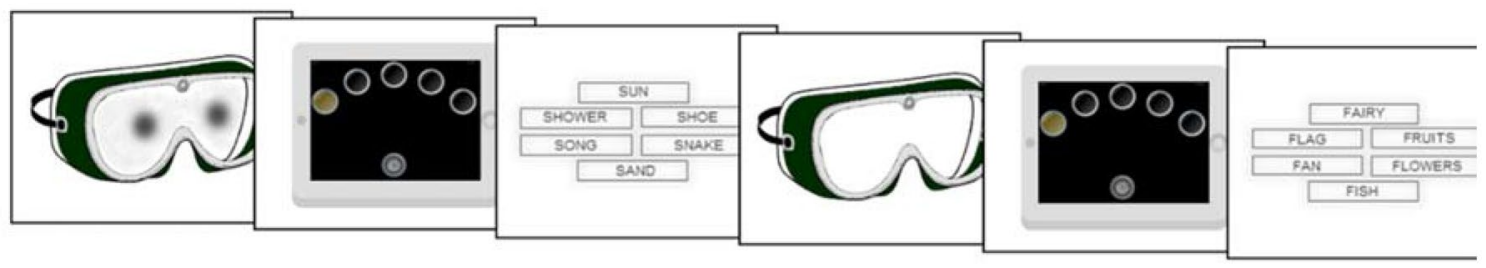

Figure 2. Participants completed the reaction time task and verbal fluency test under their assigned vision condition (normal or simulated AMD), then repeated the tasks under the opposite vision condition.

Procedure. Participants completed each cognitive task twice, under normal and simulated AMD vision (see Fig. 2). The VFT starting letter (F or S), order of cognitive task (RTI or VFT) and order of vision condition (normal or simulated AMD) were counterbalanced across participants.

Statistical analysis. We conducted separate 2 (visual condition: normal or simulated AMD) $\times 2$ (order: normal or simulated AMD vision first) repeated measures ANOVA using jamovi ${ }^{22}$ for the mental and motor responses in the RTI, and the VFT.

The TOSTER module ${ }^{23,24}$ in jamovi with equivalence bounds of \pm 0.5 Cohen's $\mathrm{d}_{\mathrm{z}}$ and an alpha of 0.05 was used to test for equivalence between the two vision conditions in the case of non-significant results in the above analyses.

To further quantify the impact of AMD on cognitive performance, the cNORMJ module ${ }^{25}$ in jamovi estimated T-scores based on the results of the normal vision condition. Using an inverted ranking order, a quartic polynomial regression modelled the relationship between raw and norm scores. A norm table for normal vision was compiled based on the model, and changes in percentile ranks for the simulated AMD condition was calculated.

\section{Results}

For the RTI, mental response times significantly increased in the simulated AMD condition (381.98, $\mathrm{SD}=29.90 \mathrm{~ms})$ compared to the normal vision condition $(359.02, \mathrm{SD}=28.04 \mathrm{~ms}$; see Fig. 3$),(F(1,22)=31.66$, $\left.p<0.001, n^{2}{ }_{\mathrm{p}}=0.59\right)$. There was no main effect of order $\left(F(1,22)=1.01, p=0.33, n_{\mathrm{p}}^{2}=0.04\right)$ or an interaction between vision and order $\left(F(1,22)=0.315, p=0.58, n_{p}^{2}=0.01\right)$.

For the RTI motor responses, there was also a main effect of vision $\left(F(1,22)=9.65, p=0.005, n^{2}{ }_{p}=0.30\right)$, with slower movement times in the $\operatorname{AMD}(265.67, \mathrm{SD}=61.79 \mathrm{~ms})$ compared to the normal vision $(250.56$, $\mathrm{SD}=52.87 \mathrm{~ms})$ condition. There was no main effect of order $\left(F(1,22)=0.88, p=0.359, n_{\mathrm{p}}^{2}=0.04\right)$, but a significant interaction between vision and $\operatorname{order}\left(\mathrm{F}(1,22)=8.07, p=0.01, n_{\mathrm{p}}^{2}=0.27\right)$. Simple main effects revealed no difference between vision conditions if participants started with normal vision $(p=0.859)$. However, if they completed the RTI first with the AMD goggles, their motor responses were significantly faster when they subsequently did the task with normal vision $(p<0.001)$.

In the VFT, there were no main effects of vision $\left(\mathrm{F}(1,22)=0.079, p=0.78, n_{\mathrm{p}}^{2}=0.004\right)$, order $(\mathrm{F}(1,22)=1.21$, $\left.p=0.28, n_{\mathrm{p}}^{2}=0.05\right)$, nor an interaction $\left(\mathrm{F}(1,22)=1.40, p=0.25, n_{\mathrm{p}}^{2}=0.06\right)$. Equivalence testing confirmed there were no meaningful differences in the VFT as a function of visual condition $(t(23)=2.17, p=0.017)$.

\section{Discussion}

Our findings provide a compelling demonstration of how visual impairments may significantly impact performance on cognitive tasks that rely on vision. The RTI was compromised due to the AMD simulation, yet the VFT remained unaffected. To put the findings into the context of standardized scores, the mean mental response time for the simulated AMD condition in the RTI was approximately 25 percentile ranks lower than in the normal vision condition. Being scored in the 25 th percentile instead of the 50 th percentile, as in our study, is a significant reminder to researchers that the added interference due to vision loss deserves attention and should not be easily discounted ${ }^{26}$.

Even though cognitive tests are just one aspect of the diagnostic process, the inaccurate scoring of cognitive performance could still contribute towards the misdiagnosis of cognitive related problems, including mild cognitive impairment (MCI) or dementia. In this event, subsequent issues can arise. For example, a mistaken diagnosis of dementia may precipitate unnecessary changes to a person's living, working, financial or social circumstances ${ }^{27}$. Furthermore, the diagnosis of MCI can trigger psychological problems (e.g., depression and anxiety) due to the stigma of cognitive impairment ${ }^{28}$. For people with AMD, who are already experiencing 


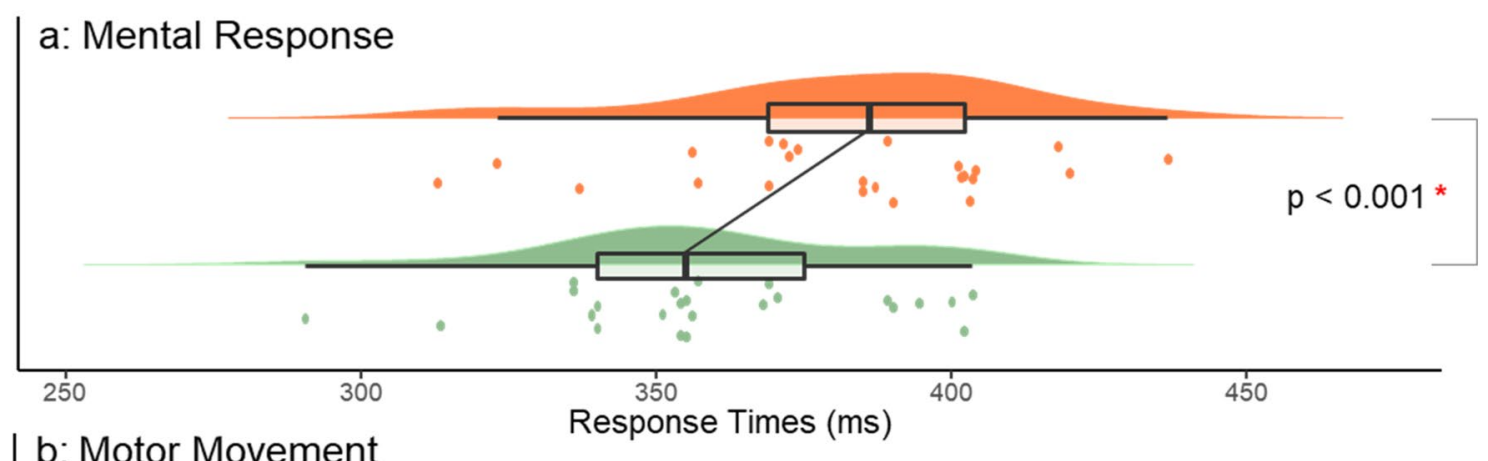

b: Motor Movement

Response Times (ms)

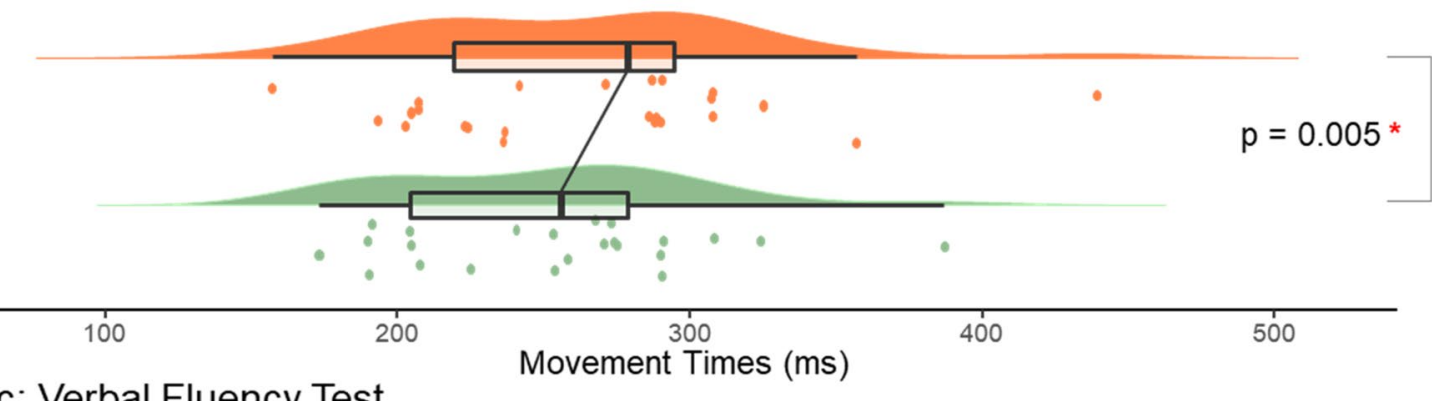

\section{c: Verbal Fluency Test}

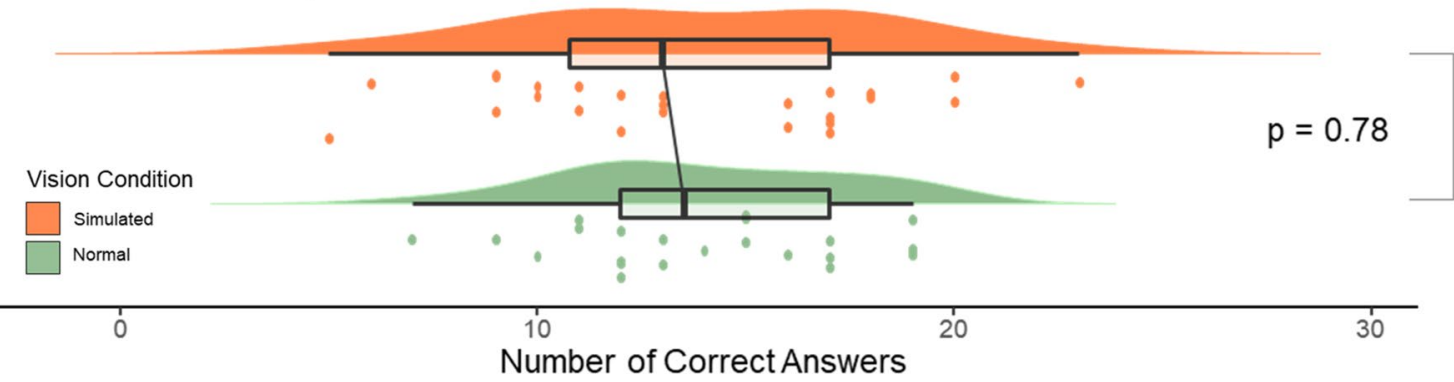

Figure 3. Normal and simulated AMD vision results. (a) RTI mental responses. Main effect of vision $(p<0.001)$. (b) RTI motor movements. Main effect of vision $(p=0.005)$. Interaction between vision and order $(p=0.01)$. (c) VFT correct answers. Main effect of vision $(p=0.78)$. Significance level: $p<0.05$.

physical and psychological issues due to vision loss ${ }^{6,29}$, the multitude of repercussions that inaccurate cognitive assessments causes are an unneeded additional burden.

It only takes the incorporation of simple precautionary measures in order to make allowances for the potential impact of AMD. For example, screening participants with mobile vision charts (e.g., Snellen) ${ }^{30}$ prior to participation, or administering vision-friendly variations of standard cognitive assessments (e.g., blind MOCA) ${ }^{31}$. While our findings specifically relate to AMD, the differences between normal and simulated conditions corroborate previous studies using paper-and-pencil tests under low visual acuity or cataract simulations ${ }^{10,11,32}$. The findings also align with studies assessing cognition in older clinical populations, indicating that this problem is systematic across a range of visual impairments ${ }^{33,34}$.

It is currently unclear whether these simulations lead to an over- or underestimation of the true impact of visual impairments on test performance, but there are reports that the severity of AMD health effects are underestimated with lenses that simulate $\mathrm{AMD}^{35}$. While the true impact of AMD on cognitive test scores remains to be established, it is clear that not controlling for vision can adversely affect the results and can have broader implications for the health of visually impaired people.

\section{Data availability}

The datasets generated during and/or analysed during the current study are available from the corresponding author on reasonable request.

Received: 1 November 2021; Accepted: 21 February 2022

Published online: 08 March 2022

\section{References}

1. American Psychological Association. Ethical principles of psychologists and code of conduct (2002, amended effective June 1, 2010, and January 1, 2017), https://www.apa.org/ethics/code (2017). 
2. Chou, R., Dana, T., Bougatsos, C., Grusing, S. \& Blazina, I. Screening for impaired visual acuity in older adults: Updated evidence report and systematic review for the US preventive services task force. JAMA 315, 915-933. https://doi.org/10.1001/jama.2016. $0783(2016)$.

3. Evans, B. J. \& Rowlands, G. Correctable visual impairment in older people: A major unmet need. Ophthalmic. Physiol. Opt. 24, 161-180. https://doi.org/10.1111/j.1475-1313.2004.00197.x (2004).

4. Bourne, R. \& GBD 2019 Blindness and Vision Impairment Collaborators; Vision Loss Expert Group of the Global Burden of Disease Study. Trends in prevalence of blindness and distance and near vision impairment over 30 years: an analysis for the Global Burden of Disease Study. Lancet Glob. Health 9, el30-e143. https://doi.org/10.1016/s2214-109x(20)30425-3 (2021).

5. Wong, W. L. et al. Global prevalence of age-related macular degeneration and disease burden projection for 2020 and 2040: A systematic review and meta-analysis. Lancet Glob. Health 2, e106-116. https://doi.org/10.1016/S2214-109X(13)70145-1 (2014).

6. Mitchell, P., Liew, G., Gopinath, B. \& Wong, T. Y. Age-related macular degeneration. Lancet 392, 1147-1159. https://doi.org/10. 1016/s0140-6736(18)31550-2 (2018).

7. Neely, D. C. et al. Prevalence of undiagnosed age-related macular degeneration in primary eye care. JAMA Ophthalmol. 135, 570-575. https://doi.org/10.1001/jamaophthalmol.2017.0830 (2017).

8. Court, H., McLean, G., Guthrie, B., Mercer, S. W. \& Smith, D. J. Visual impairment is associated with physical and mental comorbidities in older adults: A cross-sectional study. BMC Med. 12, 181. https://doi.org/10.1186/s12916-014-0181-7 (2014).

9. Macnamara, A., Chen, C., Schinazi, V. R., Saredakis, D. \& Loetscher, T. Simulating macular degeneration to investigate activities of daily living: A systematic review. Front. Neurosci. 15, 663062. https://doi.org/10.3389/fnins.2021.663062 (2021).

10. de Haan, G. A., Tucha, O. \& Heutink, J. Effects of low visual acuity on neuropsychological test scores: A simulation study. Clin. Neuropsychol. 34, 140-157. https://doi.org/10.1080/13854046.2019.1596315 (2020).

11. Wood, J. et al. Simulated visual impairment leads to cognitive slowing in older adults. Optom. Vis. Sci. 87, 1037-1043. https://doi. org/10.1097/OPX.0b013e3181fe64d7 (2010).

12. Faul, F., Erdfelder, E., Buchner, A. \& Lang, A. G. Statistical power analyses using G*Power 3.1: Tests for correlation and regression analyses. Behav. Res. Methods 41, 1149-1160. https://doi.org/10.3758/brm.41.4.1149 (2009).

13. Kanzler, C. M., Barth, J., Klucken, J. \& Eskofier, B. M. Inertial sensor based gait analysis discriminates subjects with and without visual impairment caused by simulated macular degeneration. Annu. Int. Conf. IEEE Eng. Med. Biol. Soc. 2016, 4979-4982. https:// doi.org/10.1109/embc.2016.7591845 (2016).

14. Fork in the Road Vision Rehabilitation Services. Low Vision Simulators https://www.lowvisionsimulators.com/products/macul ar-degeneration-simulators?variant $=16992106381381$ (2019).

15. Bourne, R. R. et al. Causes of vision loss worldwide, 1990-2010: a systematic analysis. Lancet Glob. Health 1, e339-349. https://doi. org/10.1016/S2214-109X(13)70113-X (2013).

16. CANTAB [Cognitive assessment software]. Cambridge Cognition, 2021).

17. Patterson, J. in Encyclopedia of Clinical Neuropsychology (eds Jeffrey S. Kreutzer, John DeLuca, \& Bruce Caplan) 1024-1026 (Springer, New York, 2011).

18. Egerhazi, A., Berecz, R., Bartok, E. \& Degrell, I. Automated neuropsychological test battery (CANTAB) in mild cognitive impairment and in Alzheimer's disease. Prog. Neuropsychopharmacol. Biol. Psychiatry 31, 746-751. https://doi.org/10.1016/j.pnpbp.2007. $01.011(2007)$.

19. Giedraitiene, N. \& Kaubrys, G. Distinctive pattern of cognitive disorders during multiple sclerosis relapse and recovery based on computerized CANTAB tests. Front. Neurol. 10, 572. https://doi.org/10.3389/fneur.2019.00572 (2019).

20. Troyer, A. K., Moscovitch, M., Winocur, G., Leach, L. \& Freedman, M. Clustering and switching on verbal fluency tests in Alzheimer's and Parkinson's disease. J. Int. Neuropsychol. Soc. 4, 137-143. https://doi.org/10.1017/s1355617798001374 (1998).

21. Vaughan, R. M., Coen, R. F., Kenny, R. \& Lawlor, B. A. Semantic and phonemic verbal fluency discrepancy in mild cognitive impairment: Potential predictor of progression to Alzheimer's disease. J. Am. Geriatr. Soc. 66, 755-759. https://doi.org/10.1111/ jgs.15294 (2018).

22. The Jamovi Project. jamovi (Version 1.6.3) [Computer Software], 2020).

23. Lakens, D. Equivalence tests: A practical primer for $t$ tests, correlations, and meta-analyses. Soc. Psychol. Personal Sci. 8, 355-362. https://doi.org/10.1177/1948550617697177 (2017).

24. Lakens, D., Scheel, A. M. \& Isager, P. M. Equivalence testing for psychological research: a tutorial. Adv. Methods Pract. Psychol. Sci. 1, 259-269. https://doi.org/10.1177/2515245918770963 (2018).

25. Lenhard, A., Lenhard, W. \& Gary, S. Continuous norming of psychometric tests: a simulation study of parametric and semiparametric approaches. PLOS ONE 14, e0222279. https://doi.org/10.1371/journal.pone.0222279 (2019).

26. Macnamara, A., Schinazi, V. R., Chen, C., Coussens, S. \& Loetscher, T. Vision impairments reduce cognitive test performance. Nat. Aging 1, 975-976 (2021).

27. Howard, R. \& Schott, J. M. When dementia is misdiagnosed. Int. J. Geriatr. Psychiatry 36, 799-801. https://doi.org/10.1002/gps. 5538 (2021).

28. Maki, Y. Reconsidering the overdiagnosis of mild cognitive impairment for dementia prevention among adults aged $\geq 80$ years. J. Prim. Health Care 13, 112-115. https://doi.org/10.1071/HC20115 (2021).

29. Taylor, D. J., Hobby, A. E., Binns, A. M. \& Crabb, D. P. How does age-related macular degeneration affect real-world visual ability and quality of life? A systematic review. BMJ Open 6, e011504. https://doi.org/10.1136/bmjopen-2016-011504 (2016).

30. Samanta, A., Mauntana, S., Barsi, Z., Yarlagadda, B. \& Nelson, P. C. Is your vision blurry? A systematic review of home-based visual acuity for telemedicine. J. Telemed. Telecare https://doi.org/10.1177/1357633x20970398 (2020).

31. Wittich, W., Phillips, N., Nasreddine, Z. S. \& Chertkow, H. Sensitivity and specificity of the montreal cognitive assessment modified for individuals who are visually impaired. J. Vis. Impair. Blind. 104, 360-368. https://doi.org/10.1177/0145482x1010400606 (2010).

32. Bertone, A., Bettinelli, L. \& Faubert, J. The impact of blurred vision on cognitive assessment. J. Clin. Exp. Neuropsychol. 29, 467-476. https://doi.org/10.1080/13803390600770793 (2007).

33. Killen, A. et al. The assessment of cognition in visually impaired older adults. Age Ageing 42, 98-102. https://doi.org/10.1093/ ageing/afs157 (2012).

34. Jefferis, J. M. et al. The impact of visual impairment on mini-mental state examination scores in the Newcastle $85+$ study. Age Ageing 41, 565-568. https://doi.org/10.1093/ageing/afs042 (2012).

35. Butt, T., Crossland, M. D., West, P., Orr, S. W. \& Rubin, G. S. Simulation contact lenses for AMD health state utility values in NICE appraisals: A different reality. Br. J. Ophthalmol. 99, 540-544. https://doi.org/10.1136/bjophthalmol-2014-305802 (2015).

\section{Acknowledgements}

A.M. was supported by the Australian Government Research Training Program Scholarship and T.L. was funded by a National Health and Medical Research Council (NHMRC) Dementia Research Leadership Fellowship (GNT1136269). 


\section{Author contributions}

A.M. and T.L. conceptualized the study. A.M. conducted the experiment and analysed the data. All authors contributed to the article and approved the submitted version.

\section{Competing interests}

The authors declare no competing interests.

\section{Additional information}

Correspondence and requests for materials should be addressed to A.M.

Reprints and permissions information is available at www.nature.com/reprints.

Publisher's note Springer Nature remains neutral with regard to jurisdictional claims in published maps and institutional affiliations.

(c) (i) Open Access This article is licensed under a Creative Commons Attribution 4.0 International License, which permits use, sharing, adaptation, distribution and reproduction in any medium or format, as long as you give appropriate credit to the original author(s) and the source, provide a link to the Creative Commons licence, and indicate if changes were made. The images or other third party material in this article are included in the article's Creative Commons licence, unless indicated otherwise in a credit line to the material. If material is not included in the article's Creative Commons licence and your intended use is not permitted by statutory regulation or exceeds the permitted use, you will need to obtain permission directly from the copyright holder. To view a copy of this licence, visit http://creativecommons.org/licenses/by/4.0/.

(C) The Author(s) 2022 